\title{
Effect of L-citrulline, L-leucine, and multicomponent exercises on body compositions, physical activity, and amino acid concentrations in older Japanese women with low body mass index: A randomized double-blind placebo-controlled study
}

\section{Mijin Kim ( $\nabla$ mjmj0816@gmail.com )}

University of Tsukuba Faculty of Health and Sport Sciences: Tsukuba Daigaku Taiikukei https://orcid.org/0000-0002-7216-7112

\section{Hiroko Isoda}

University of Tsukuba Graduate School of Life and Environmental Sciences: Tsukuba Daigaku Daigakuin Seimei Kankyo Kagaku Kenkyuka

\section{Tomohiro Okura}

University of Tsukuba Faculty of Health and Sport Sciences: Tsukuba Daigaku Taiikukei

\section{Research article}

Keywords: Sarcopenia, Leucine, Citrulline, Weight-bearing exercise, Older adults, Amino acids

Posted Date: March 13th, 2021

DOl: https://doi.org/10.21203/rs.3.rs-311313/v1

License: (c) (i) This work is licensed under a Creative Commons Attribution 4.0 International License. Read Full License 


\section{Abstract}

Background: The intake of citrulline (CIT) and leucine (LEU) can stimulate protein synthesis. However, the efficacy of the combined intervention of CIT and LEU intake with exercise on body composition and physical activity (PA) remains unclear. This study aimed to investigate the combined effect of CIT and LEU intake and weight-bearing exercises (WBE) and square stepping exercise (SSE) for 20 weeks on body composition, PA, and amino acid concentration in older women with low body mass index (BMI) (16 to 21 $\mathrm{kg} / \mathrm{m} 2$ ). Methods: A total of 23 participants practiced WBE and SSE once a week for 75 minutes and were administered supplement (Ex + CIT·LEU group: CIT $0.8 \mathrm{~g}$ and LEU $1.6 \mathrm{~g}$; Ex + Placebo group: $3.5 \mathrm{~g}$ carbohydrate) twice a day for 20 weeks. Body composition was measured using dual-energy X-ray absorptiometry. PA, including leisure-time, household, and occupational PA, was assessed using the Physical Activity Scale for the Elderly. Amino acid concentrations in the blood were analyzed by highperformance liquid chromatography.

Results: Significant interactions were observed in the body weight, BMI, lean mass, body mass, household and total PA, tyrosine, and phenylalanine. The within-group analysis showed that tyrosine of postintervention measurement (Post) significantly in both groups $(p<0.05)$. Body weight, BMl, lean mass, body mass, household PA, total PA, and phenylalanine of Post increased significantly in the Ex + CIT·LEU group ( $p<0.05$ ). Additionally, significant positive correlations were observed between the intake rate of supplements and bone mass $(r=0.80)$ and between the practice rate of WBE at home and bone mineral density $(r=0.66)$ in the Ex + CIT·LEU group.

Conclusion: Our findings suggest that the intake of CIT and LEU, with the practice of WBE and SSE, could improve body weight, muscle mass, bone mass, and PA in older women with low BMI, which may prevent sarcopenia and frailty.

Trial registration: UMIN000022385. Registered 20 May 2016, https://upload.umin.ac.jp/cgi-open-39 $\mathrm{bin} / \mathrm{ctr} / \mathrm{ctr}$.cgi?function=brows\&action=brows\&recptno=R000025797\&type=summary\&language $=\mathrm{J}$

\section{Full Text}

Due to technical limitations, full-text HTML conversion of this manuscript could not be completed. However, the manuscript can be downloaded and accessed as a PDF.

\section{Figures}




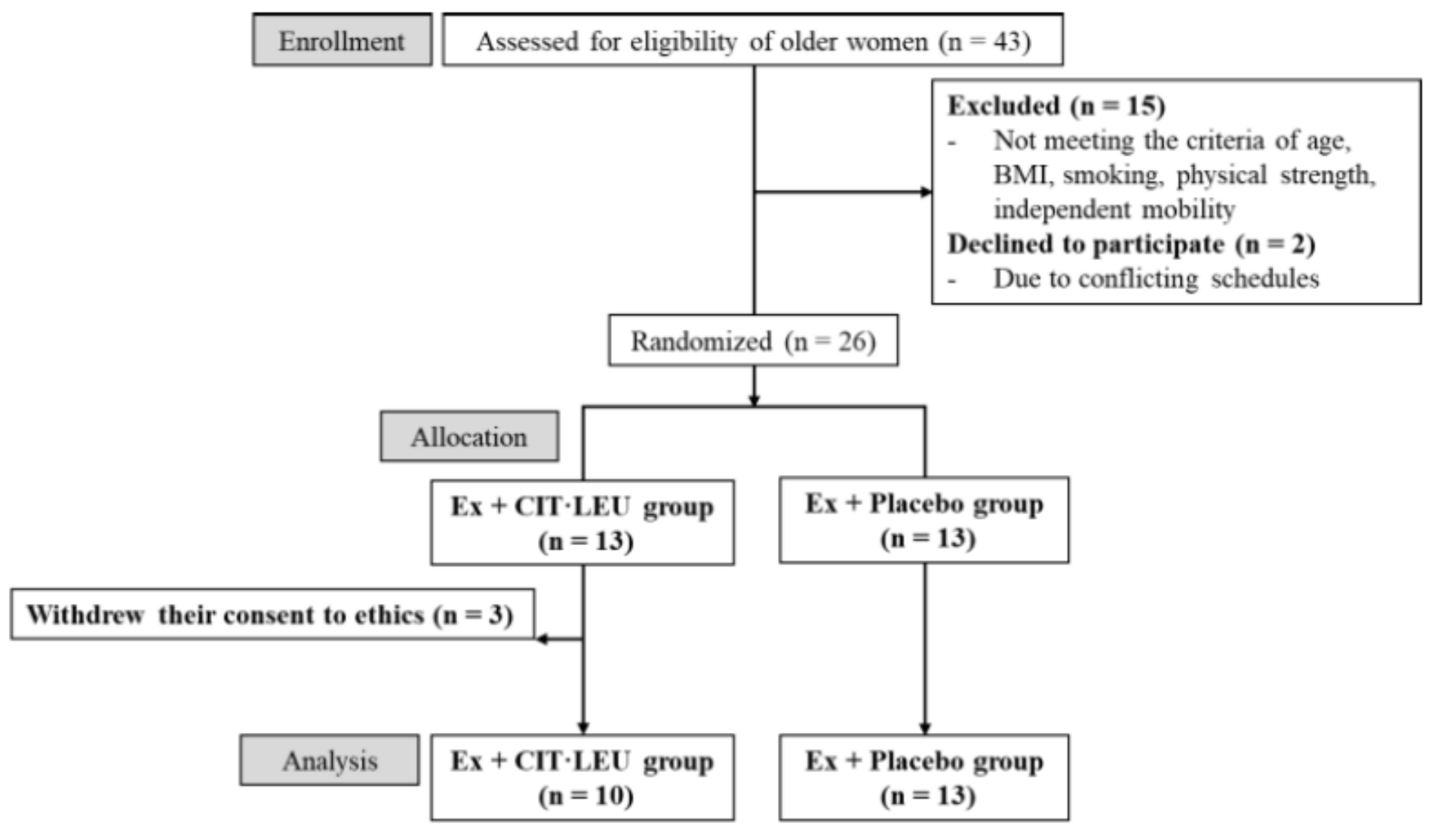

Figure 1

Flow-chart diagram 